\title{
(6) OPEN ACCESS \\ Twelve-month analgesia and rescue, by cooled radiofrequency ablation treatment of osteoarthritic knee pain: results from a prospective, multicenter, randomized, cross-over trial
}

\author{
Tim Davis, ${ }^{1}$ Eric Loudermilk, ${ }^{2}$ Michael DePalma, ${ }^{3}$ Corey Hunter, ${ }^{4}$ David A Lindley, ${ }^{5}$ \\ Nileshkumar Patel, ${ }^{6}$ Daniel Choi, ${ }^{7}$ Marc Soloman, ${ }^{8}$ Anita Gupta, ${ }^{9}$ Mehul Desai, ${ }_{1}^{10}$ \\ Elizabeth Cook, ${ }^{6}$ Leonardo Kapural ${ }^{11}$
}

${ }^{1}$ Orthopedic Pain Specialists, Santa Monica, California, USA 2Piedmont Comprehensive Pain Management Group, Greenville, South Carolina, USA

${ }^{3}$ Virginia iSpine Physicians, Richmond, Virginia, USA

${ }^{4}$ Ainsworth Institute of Pain, New York City, New York, USA 5 Interventional Pain

Management of Texas, Mineral Wells, Texas, USA

${ }^{6}$ Advanced Pain Management, Greenfield, Wisconsin, USA

${ }^{7}$ Valley Pain Consultants, Scottsdale, Arizona, USA

${ }^{8}$ Valley Anesthesia Consultants, Phoenix, Arizona, USA

${ }^{9}$ Drexel University, Philadelphia, Pennsylvania, USA

${ }^{10}$ International Spine, Pain

\& Performance Center

Washington, Washington DC, USA

${ }^{11}$ Center for Clinical Research, Winston Salem, North Carolina, USA

\section{Correspondence to} Dr Leonardo Kapural, Center for Clinical Research, Winston Salem, NC 27103, USA: Ikapural@ccrpain.com

Received 19 August 2018 Revised 15 December 2018 Accepted 21 January 2019

Published Online First

16 February 2019

Check for updates

(C) American Society of Regional Anesthesia \& Pain Medicine 2019. Re-use permitted under CC BY-NC. No commercial re-use. Published by BMJ.

\section{To cite: Davis T,}

Loudermilk E, DePalma M,

et al. Reg Anesth Pain Med

2019:44:499-506.

\section{ABSTRACT}

Background and objectives As a follow-up to the 6 -month report, ${ }^{12}$ this study investigated the analgesic effect of cooled radiofrequency ablation (CRFA) in patients with knee osteoarthritis (OA) 12 months postintervention and its ability to provide pain relief in patients who experienced unsatisfactory effects of intraarticular steroid injection (IAS).

Methods Seventy-eight per cent (52/67) of patients originally treated with CRFA were evaluated at 12 months, while at 6 months post-IAS, 82\% (58/71) of those patients crossed over to CRFA and assessed 6 months later.

Results At 12 months, 65\% of the original CRFA group had pain reduction $\geq 50 \%$, and the mean overall drop was 4.3 points $(p<0.0001)$ on the numeric rating scale. Seventy-five per cent reported 'improved' effects. The cross-over group demonstrated improvements in pain and functional capacity $(p<0.0001)$. No unanticipated adverse events occurred.

Conclusions This study demonstrates that analgesia following CRFA for OA knee pain could last for at least 12 months and could rescue patients who continue to experience intolerable discomfort following IAS.

Clinical trial registration The ClinicalTrials.gov registration number for this study is NCT02343003.

\section{INTRODUCTION}

Total knee arthroplasty (TKA) is an effective therapeutic option of last resort for individuals afflicted with significant osteoarthritis (OA)-related knee pain and dysfunction. While the outcomes of TKA are consistent and well established, ${ }^{12}$ the procedure may not be indicated in patients who have comorbidities, ${ }^{3}$ or those who otherwise may not be appropriate candidates for TKA. ${ }^{4}$ Therefore, providing a therapeutic option with long-term duration of effect may enable such patients to have a more satisfactory quality-of-life.

The minimally invasive, outpatient nature of radiofrequency ablation (RFA) of targeted nociceptive nerves is becoming an increasingly well known and timely option for patients in whom conservative therapies have failed and/or those who are not candidates for TKA. ${ }^{5-8}$ In particular, the 'cooled' form of RFA (CRFA) has afforded patients with knee OA with pain relief ${ }^{9-12}$ and functional improvement. $^{9} 1112$ Most recently, we reported that $74 \%$ of patients treated with CRFA had pain reduction of $50 \%$ or more compared with $16 \%$ of demographically matched patients who received an intra-articular steroid injection (IAS) at 6 months postintervention. ${ }^{12}$ In addition, through secondary measures, significantly more patients at 6 months reported 'satisfactory joint function' via the Oxford Knee Score and a perception that their treatment effect had 'improved' their condition than those who received an IAS per the Patient Global Perceived Effect. Although the beneficial effectiveness of CRFA for treating OA of the knee was evident from the 6-month analyses of this study, ${ }^{12}$ a paucity of data has been published regarding longer term durability of these effects.

This analysis explored the sustainability of analgesic effects realized at 6 months in patients with knee OA who were treated with CRFA. We hypothesized that significant $(\geq 50 \%)$ analgesia would remain among the majority $(>50 \%)$ of patients in the original CRFA group 12 months postintervention and that patients who still had intolerable discomfort 6 months following IAS would experience significant $(\geq 50 \%)$ pain relief after CRFA. As such, this current study primarily evaluated the proportion of patients whose knee pain was reduced by $\geq 50 \%$ from baseline 12 months post-treatment within the initial cohort of patients with OA who were enrolled in the 6-month clinical trial at 11 different sites. ${ }^{12}$ Additionally, clinical features of subjects who elected to cross-over to receive CRFA after 6 months ('cross-over' (XO) group) were evaluated.

\section{METHODS}

All patients were properly consented prior to initiating screening activities. The study is registered in ClinicalTrials.gov: registration number, NCT02343003; initial release date, 15 January 2015.

\section{Study design}

This prospective, randomized, open-label, multicenter (11 sites) clinical study with a parallel-group design initially included the test treatment, 
CRFA ( $\mathrm{N}=76$ ), utilizing the Coolief System (Halyard Health, Alpharetta, Georgia, USA), or IAS $(\mathrm{N}=75)$, in a $1: 1$ randomization scheme. The methodological differences between the active treatment comparators in this study did not permit blinding of investigators or patients to the interventions. The initial results from this study presented data through study follow-up visits at 1,3 , and 6 months compared with the two study groups primarily by the proportion of subjects whose knee pain was reduced by $\geq 50 \%$ from baseline at 6 months post-treatment. Additional secondary measures noted improvements in function, and nearly all patients in the CRFA group (91\%) reported perceptions of 'improvement' regarding their knee pain. ${ }^{12}$ The focus of this report is to describe the patient's experience through 12 months. Additionally, to further evaluate CRFA, patients who were dissatisfied with their IAS treatment after 6 months could cross-over to the ablation treatment. The substantial migration of original IAS study group members to the XO group left only four patients in the former cohort, which was considered too small to conduct any meaningful analytical statistical comparisons between CRFA and IAS treatments at 12 months post-interventions. Patients in the IAS cohort who elected to receive CRFA treatment at the 6-month follow-up visit were followed for an additional 6 months and are herein referred to as XO group members. Methodology, patient demographics, and 6-month results for the original CRFA and IAS study groups have been published. ${ }^{12}$

\section{Study population}

Patients who had radiographic evidence of OA within 12 months prior to study screening, with no other etiology demonstrated as the source of knee pain, were eligible for the study. While individuals with bilateral knee OA were not excluded; only one knee was screened and enrolled as the 'index knee' for treatment. Management of contralateral knee pain in bilateral patients was left up to the discretion of the investigators and patients as part of standard of care. Selection criteria included: knee pain $\geq 6$ months that was unresponsive to conservative treatments (physical therapy, oral analgesics: $\leq 60 \mathrm{mg}$ morphine equivalence, stable for 2 months; intra-articular injections with steroids and/ or viscosupplementation), body mass index (BMI) $<40$, and reporting $\geq 50 \%$ response to blocks as described previously ${ }^{12}$ and below. On confirmation that a patient was eligible, randomization was completed utilizing prepopulated, sequentially numbered, sealed envelopes generated by the statistician using a computerized randomization programme. Sites opened a single envelope per patient and chose the lowest available number to maintain sequential ordering of randomization. Additional treatments for the index knee were prohibited during the study.

The block paradigm was as follows: patients who indicated a score reduction on the numeric rating scale (NRS) $\geq 50 \%{ }^{5}$ at least 15 min following fluoroscopically guided blockade of the superomedial and inferomedial branches of the saphenous nerve and the superolateral branch of the femoral nerve ${ }^{13}$ using $0.60-$ $0.75 \mathrm{~mL} / \mathrm{site}$ of local anesthetic (preferably Marcaine (bupivacaine) Hospira, Lake Forest, Illinois, USA, $0.5 \%$ or similar) at each site were eligible for the study. ${ }^{12}$

\section{Study intervention}

Cooled RFA of the index knee was administered to patients in the CRFA study cohort, as facilitated by fluoroscopic visualization of anatomical landmarks. ${ }^{13}$ A 75 or $100 \mathrm{~mm}$ 17-gage CRF introducer was placed at the appropriate locations after $1-3 \mathrm{~mL}$ of $1 \%$ lidocaine was infiltrated. An 18-gage internally cooled 4 $\mathrm{mm}$ active tip electrode was placed into the introducer needle, and $50 \mathrm{~Hz}$ sensory stimulation at $<0.5 \mathrm{~V}$ in all three locations reproduced concordant knee pain that ensured proximity of the probe to each of the target nerves (superomedial and inferomedial branches of the saphenous nerve and the superolateral branch of the femoral nerve ${ }^{13}$ prior to lesioning. Next, motor stimulation at $2 \mathrm{~Hz}$ was carried on up to $1 \mathrm{~V}$ without muscular contractions to ensure proper distance of final radiofrequency (RF) needle active tip position from any motor nerve fibers.

The CRFA intervention produces thermal energy with average maximum tissue temperatures greater than $80^{\circ} \mathrm{C},{ }^{14}$ while the probe tip temperature is maintained at $60^{\circ} \mathrm{C}$ by the cooling water circulating within the probe. Each lesion was created over $150 \mathrm{~s}$. Following the procedure and patient recovery, each patient was discharged to home with instructions to limit strenuous activity for at least 24 hours postprocedure.

\section{Study outcomes}

The proportion of subjects whose knee pain was reduced by $\geq 50 \%$ compared with baseline was calculated at 12 months post-treatment, ${ }^{15}$ as measured by the NRS. Secondary endpoints included: (1) change in knee function detected by the Oxford Knee Score (OKS) - a validated outcomes instrument that is routinely used to evaluate the overall condition of subjects with knee OA, ${ }^{16}(2)$ subjects' perception of treatment effect as reflected by the Global Perceived Effect score, and (3) opioid analgesic use, as measured by subject self-reported average daily dosage used. Reported assessments of these study endpoints were based on patients' impressions made during the week preceding data collection at each study visit for the original CRFA group (baseline and 12 months) and XO group (baseline and 6 months). The baseline values utilized for $\mathrm{XO}$ analysis were those at the time of cross-over for all outcome measures. All subjects were evaluated for adverse events (AEs) and serious AEs (SAEs) at each visit.

To investigate a theoretical concern that CRFA could inadvertently progress knee OA relative to evidence provided at study baseline, an amendment was created late in the study to allow for the collection of radiographs at 12 months. Fifty-one images were considered, and the disease state displayed by each was quantified by independent radiologists (generally) per the Kellergan-Lawrence Scale. Twenty-four images were from the originally treated CRFA group and 27 were from the XO group.

\section{Statistical analysis}

A non-inferiority evaluation was used to estimate the study sample size. The sample size was based on the estimated success rates of $59 \%{ }^{8}$ (success $\geq 50 \%$ NRS score reduction) and $47 \% 0^{17}$ (success $\geq 30 \%$ NRS score reduction) in the CRFA and standard groups, respectively, and a non-inferiority margin of $15 \%$. Assuming an attrition rate of $20 \%$ and a two-sided significance level of 5\%, 144 subjects enrolled into the study would yield 114 subjects at the primary endpoint.

As was previously reported on 6-month outcomes of this study, ${ }^{12}$ the 12 -month data are derived from the full-analysis study population set, while the $\mathrm{XO}$ results are from the per-protocol set. The protocol defined the full analysis set as: all randomized subjects will be analyzed following the principle of intention-to-treat (ITT) provided they received Coolief or corticosteroid injection treatment and had at least one effectiveness observation, thereby, the results presented can be considered a modified ITT. Percentages are reported with 95\% CI. Within-group comparisons were expressed as mean and an associated SD, with significant differences indicated by $\mathrm{p} \leq 0.05$. Such 


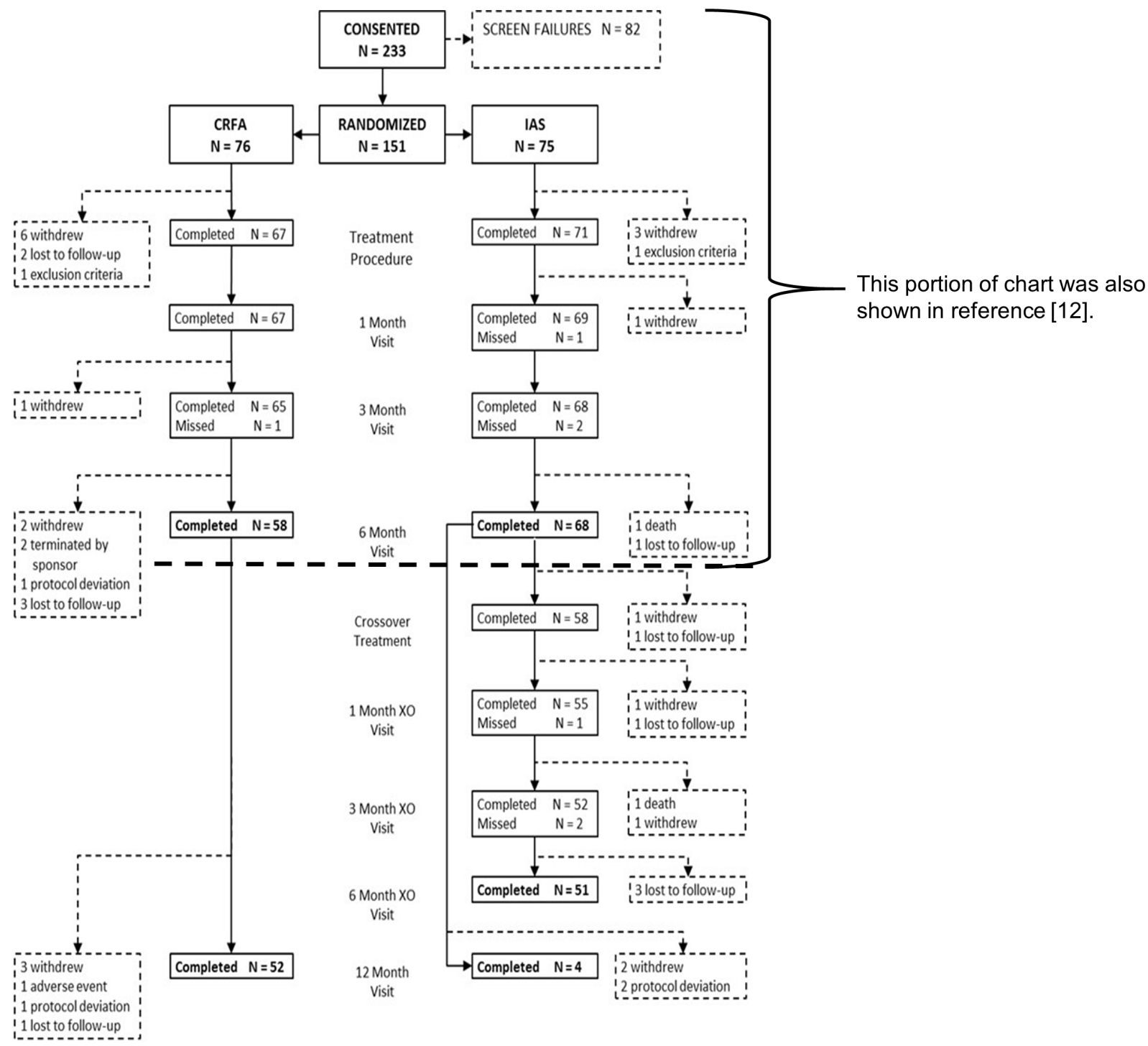

Figure 1 Consolidated Standards of Reporting Trials diagram displaying patients through study stages. *Two subjects were terminated by the sponsor, because the principal investigator changed jobs and a suitable replacement could not be identified. The site was closed, and subjects were dropped. ${ }^{*}$ Adverse event—subject had return of index knee pain and chose a surgical alternative.

analyses were not prespecified, and so no type I error adjustment for multiplicity was made to preserve the overall $5 \%$ level of significance. Assessment determinations were made from aggregates of data collections from all available patients at each study time point.

\section{RESULTS}

\section{Disposition of study patients}

Out of the 233 patients screened, 151 were enrolled into the initial study, ${ }^{12}$ with 76 and 75 randomized to the CRFA and IAS study groups, respectively ${ }^{12}$ (figure 1 ). Of those randomized, 67 patients in the CRFA and 71 patients in the IAS group were treated. ${ }^{12}$ At 6 months, 58 (87\%) and 68 (96\%) of treated patients in the CRFA and IAS cohorts contributed data to the primary endpoint, ${ }^{12}$ and $58(82 \%)$ patients of the IAS group crossed over to receive CRFA. At 12 months, 52 (78\%) patients in the originally treated CRFA group contributed data to the primary endpoint, while at 6 months post-CRFA, 51 $(88 \%)$ patients in the XO group did the same. Four patients $(6 \%)$ of the IAS group completed the 12-month visit. At the time of cross-over eligibility, three of these four patients were not in severe enough pain to warrant intervention and one did not want the procedure due to comorbid conditions.

\section{Study population}

Baseline demographic variables, including age, gender and race distributions, mean $\mathrm{BMI}$, mean duration of knee pain, analgesic medication utilization, knee OA severity, mean index knee pain levels (NRS scores) before diagnostic block, and the extent of index knee pain reduction postdiagnostic block were made available previously. ${ }^{12}$

\section{Pain assessment in the original CRFA group at 12 months}

Raw data are presented in table 1. The mean $4.3 \pm 2.7$ (SD)-point decrease in NRS from baseline at 12 months in the original CRFA group was statistically significant $(\mathrm{N}=52$, $\mathrm{p}<0.0001$, paired Student's t-test). The mean alteration in the NRS score at the 12 -month time point was similar with that reported at 6 months, where a mean improvement of $4.9 \pm 2.4$ 
Table 1 Study outcomes: original CRFA group versus IAS group up to 12 months

\begin{tabular}{|c|c|c|c|c|c|c|c|c|c|c|}
\hline & \multicolumn{10}{|c|}{ Numeric Rating Scale } \\
\hline & \multicolumn{2}{|l|}{ Baseline } & \multicolumn{2}{|l|}{1 Month } & \multicolumn{2}{|l|}{3 Months } & \multicolumn{2}{|l|}{6 Months } & \multicolumn{2}{|c|}{12 Months } \\
\hline & CRFA & IAS & CRFA & IAS & CRFA & IAS & CRFA & IAS & CRFA & IAS \\
\hline $\mathrm{N}$ & 76 & 75 & 67 & 69 & 65 & 68 & 58 & 68 & 52 & 4 \\
\hline Mean & 7.3 & 7.2 & 3.0 & 3.9 & 2.8 & 5.2 & 2.5 & 5.9 & 3.1 & 3.3 \\
\hline SD & 1.2 & 1.0 & 2.3 & 2.2 & 2.2 & 2.0 & 2.3 & 2.2 & 2.7 & 3.3 \\
\hline $\begin{array}{l}\text { P value for difference } \\
\text { between groups* }\end{array}$ & 0.55 & & 0.025 & & $<0.0001$ & & $<0.0001$ & & 0.99 & \\
\hline
\end{tabular}

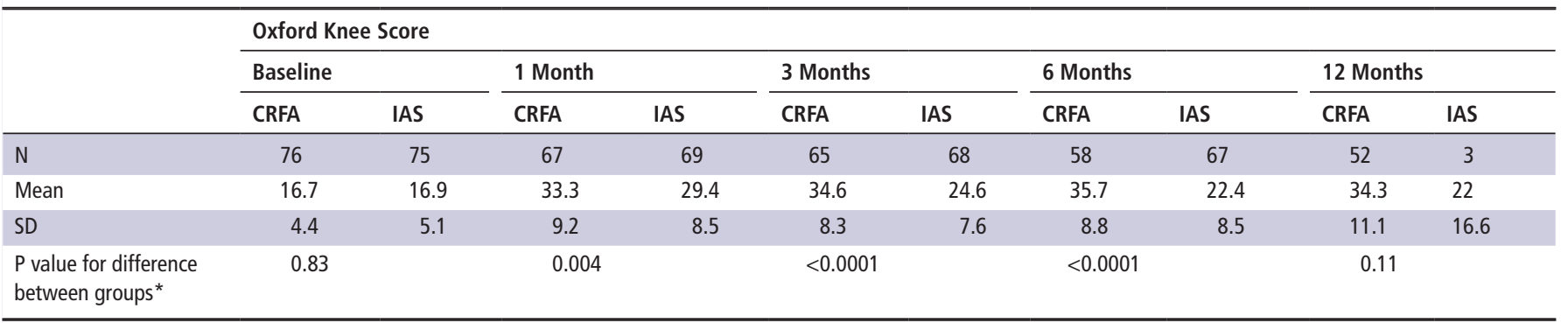

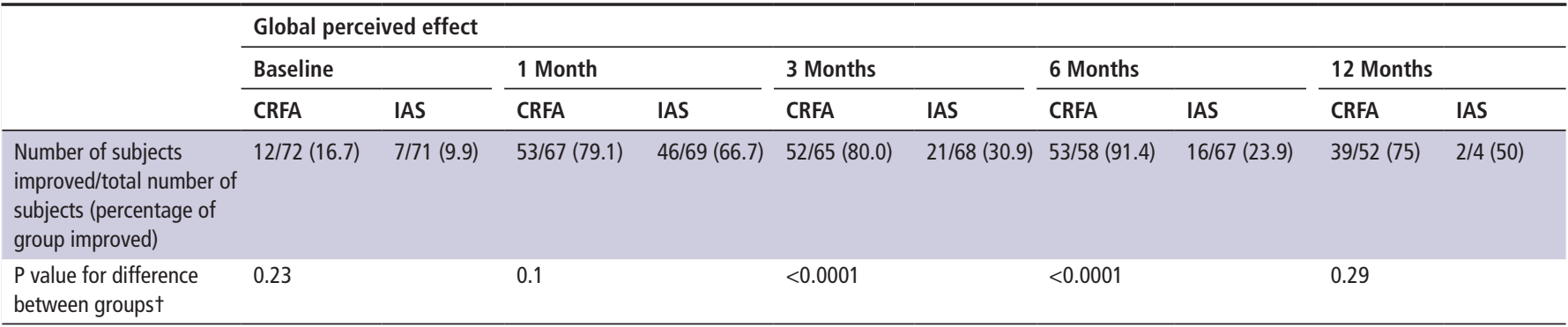

${ }^{*} \mathrm{P} \leq 0.05$ indicates a significant difference.

†Full-analysis study set data are presented.

CRFA, cooled radiofrequency ablation; IAS, intra-articular steroid; $\mathrm{N}$, number of study subjects.

points was identified. ${ }^{12}$ Further evidence for this sustained response to CRFA is shown in figure 2, where the mean NRS scores following CRFA are similar from 1 to 12 months. And whereas $70 \%$ (47/67) (95\% CI 59.2 to 81.1$)$, $72 \%(47 / 65)$ (95\% CI 61.4 to 83.2 ) and $74 \%$ (43/58) (95\% CI 62.9 to 85.4 ) of the CRFA group experienced diminished pain relative to baseline that was $\geq 50 \%$ at 1,3 , and 6 months, respectively ${ }^{12}$;

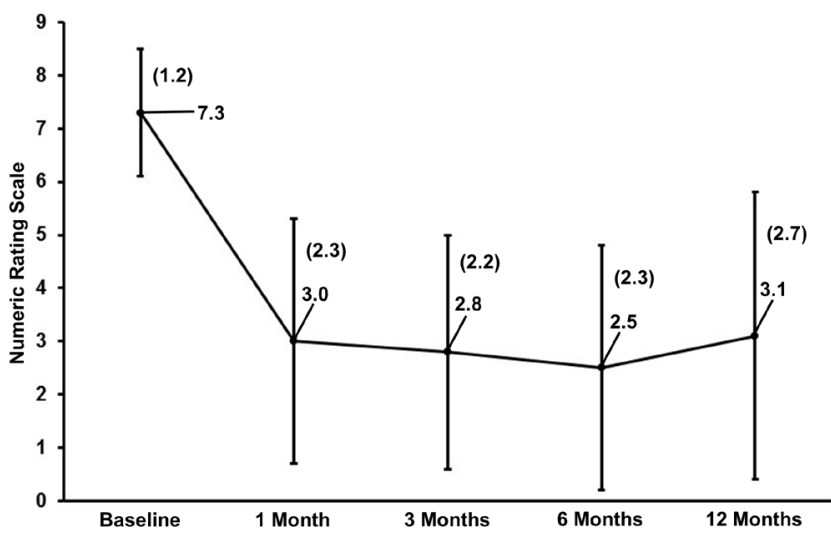

Figure 2 Mean pain scores (Numerical Rating Scale) in the cooled radiofrequency ablation cohort over time. SD are indicated in parentheses. Baseline, $\mathrm{N}=76 ; 1$ month, $\mathrm{N}=67 ; 3$ months, $\mathrm{N}=65 ; 6$ months, $\mathrm{N}=58 ; 12$ months, $\mathrm{N}=52$. Baseline through 6 -month values were previously reported. ${ }^{12}$
$65 \%(34 / 52)(95 \%$ CI 52.5 to 78.3$)$ of the group reported this clinically relevant ${ }^{15}$ outcome at 12 months.

\section{Secondary study outcomes in the original CRFA group at 12} months

At 12 months, the OKS increase from baseline in the original CRFA cohort was $17.3 \pm 12$ points $(\mathrm{N}=52, \mathrm{p}<0.0001$, Student's paired t-test), with an absolute mean of $34.3 \pm 11.1$ points. The fraction of patients in the CRFA group experiencing 'severe arthritis' and 'satisfactory knee function' (as defined by the OKS scale) with time post-treatment was inversely distributed (figure 3). Indeed, the percentage of patients reporting OKS 'severe arthritis' was progressively reduced from baseline to 6 months and was nearly sevenfold less at 12 months compared with baseline. In contrast, while there were no patients with OKS 'satisfactory joint function' in the CRFA group at baseline, the proportion of CRFA patients in this group consistently increased throughout the study, with nearly half reporting this outcome at 12 months. Patients who claimed 'moderate to severe arthritis' were approximately $25 \%$ at all time points, while those reporting 'mild to moderate' OKS were approximately $40 \%$ through 6 months, but then dropped to $17 \%$ at 12 months.

The proportion of patients in the CRFA group who had a perception of the treatment effect on their health as being 'improved' at 12 months was 75\% (39/52) (95\% CI 63.2 to $86.8)$, which was similar to values at $1(79 \%, 53 / 67)(95 \%$ CI 69.4 to 88.8$)$ and 3 months $(80 \%, 52 / 65)$ (95\% CI 70.3 to 89.7$)$ post-treatment and substantially different than the 


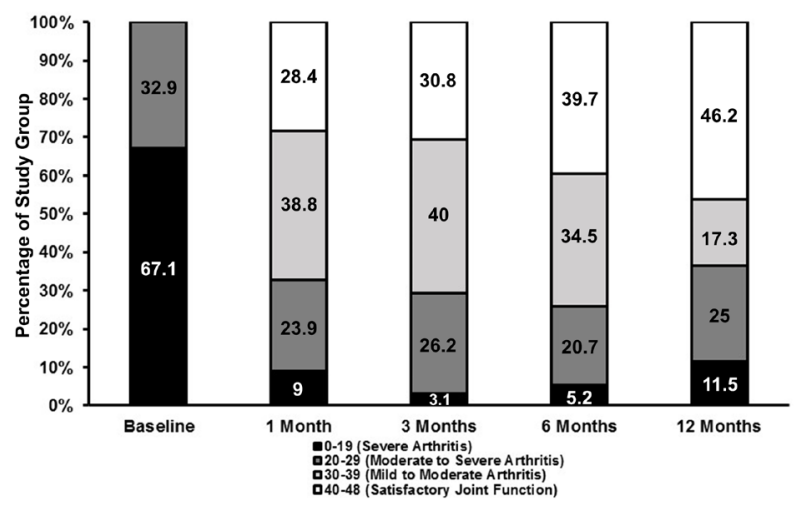

Figure 3 Knee function (Oxford Knee Scale) in the cooled radiofrequency ablation group over time. Baseline, $\mathrm{N}=76 ; 1$ month, $\mathrm{N}=67 ; 3$ months, $\mathrm{N}=65 ; 6$ months, $\mathrm{N}=58 ; 12$ months, $\mathrm{N}=52$.

baseline value of $17 \%$ (12/72) (95\% CI 8.1 to 25.3$).{ }^{12}$ Proportions for all of the aforementioned time points are less than that observed at 6 months $(91 \%, 53 / 58)(95 \%$ CI 84.2 to 98.6). ${ }^{12}$

The mean total daily dose in opioid analgesic medication (morphine equivalents in $\mathrm{mg}$ ) in the CRFA group at 12 months was $30.3 \pm 27.4 \mathrm{mg}(\mathrm{N}=17)$, which was similar to the baseline value (delta $=-1 \pm 10.3 \mathrm{mg}, \mathrm{N}=17, \mathrm{p}=0.68$, paired Student's t-test). As noted in the previous publication, $43 \%$ of patients in the CRFA group who were taking opioids as of the study's baseline assessment were using such medication for medical indications beyond OA related knee pain (ie, knee and back pain, back pain, etc). Additionally, a subgroup analysis was undertaken examining response to treatment of patients from the original CRFA group who were not taking opioids to manage their pain at study baseline. Fourteen of $67(21 \%)$ patients fell into this category and of those, 11 (79\%) patients indicated $\geq 50 \%$ relief of their baseline reported index knee pain at 6 months. This subgroup reported greater pain relief (mean NRS point reduction $=6.1$ at 6 months) than what was observed for the entire originally treated CRFA group at 6 (mean improvement $=4.9$ points) or 12 months (mean improvement $=4.3$ points $).^{12}$

Pain assessment in the $\mathrm{XO}$ group at 6 months

The XO group had significant reductions from baseline, reporting mean changes of $3.1 \pm 2.5$ points $(\mathrm{N}=40), 3.6 \pm 2.4$ points $(\mathrm{N}=38)$, and $3.2 \pm 2.7$ points $(\mathrm{N}=37)$ in the NRS at 1,3 , and 6 months, respectively $(\mathrm{p}<0.0001$, paired Student's $t$-test). These similar point reductions are reflected by the consistent NRS score means observed across the follow-up time points in this group (figure 4). Forty-nine per cent (18/37) (95\% CI 32.5 to 64.8 ) of the XO group experienced clinically relevant ${ }^{15}$ pain relief compared with baseline that was $\geq 50 \%$ at 6 months.

\section{Secondary study outcomes in the XO group at 6 months}

Improvements in function were also noted in the XO group, and the mean increase in the OKS from baseline in the $\mathrm{XO}$ group at 6 months was $11.6 \pm 9.8$ points $(\mathrm{N}=36, \mathrm{p}<0.0001$, Student's paired t-test). The mean OKS at each study time point was $18.6 \pm 6.6(\mathrm{~N}=42), 30 \pm 9.4 \quad(\mathrm{~N}=40), 30.3 \pm 10$ $(\mathrm{N}=38), 29.8 \pm 10.6(\mathrm{~N}=37)$, at baseline, 1,3 , and 6 months, respectively. While none of the XO patients reported OKS

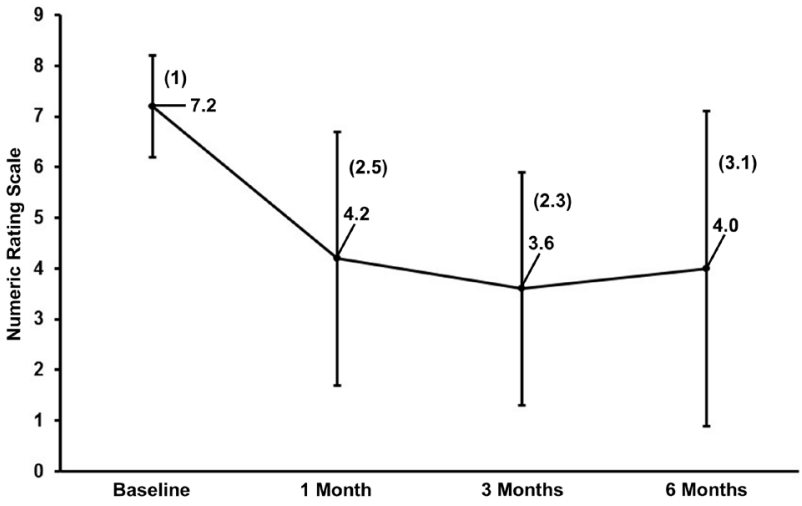

Figure 4 Mean pain scores (Numerical Rating Scale) in the cross-over group over time. SD are indicated in parentheses. Baseline, $\mathrm{N}=66 ; 1$ month, $\mathrm{N}=40 ; 3$ months, $\mathrm{N}=38 ; 6$ months, $\mathrm{N}=37$.

'satisfactory joint function' at baseline (6 months post-IAS), approximately two-thirds of XO cohort members reported OKS 'severe arthritis' at this time point (figure 5). However, nearly one-fifth of the XO group reported OKS 'satisfactory joint function' 1 month after CRFA, and this condition progressively increased to include approximately one-quarter of the cohort by 6 months. In contrast, the incidence of OKS 'severe arthritis' in the XO group fell more than fourfold at 1 month and included approximately one-fifth of the cohort at 6 months. The frequency of OKS 'moderate to severe arthritis' fell by more than $10 \%$ from 1 to 6 months post-CRFA, while the proportion of patients having OKS 'mild to moderate arthritis' consistently remained at approximately 34\% during this time frame.

While at the baseline (6 months post-IAS), 7.1\% (3/42) (95\% CI 0.0 to 14.9 ) of XO group members described the effect of CRFA on their health as 'improved', 65\% (26/40) (95\% CI 50.2 to 79.8 ), 79\% (30/38) (95\% CI 66.0 to 91.9 ), and $57 \%(21 / 37)$ (95\% CI 40.8 to 72.7$)$ of the group reported this outcome at 1,3 , and 6 months post-CRFA, respectively.

\section{Radiographic evidence of knee OA}

To understand the state of knee OA following CRFA, an amendment was added late in the study to collect $\mathrm{x}$-rays at

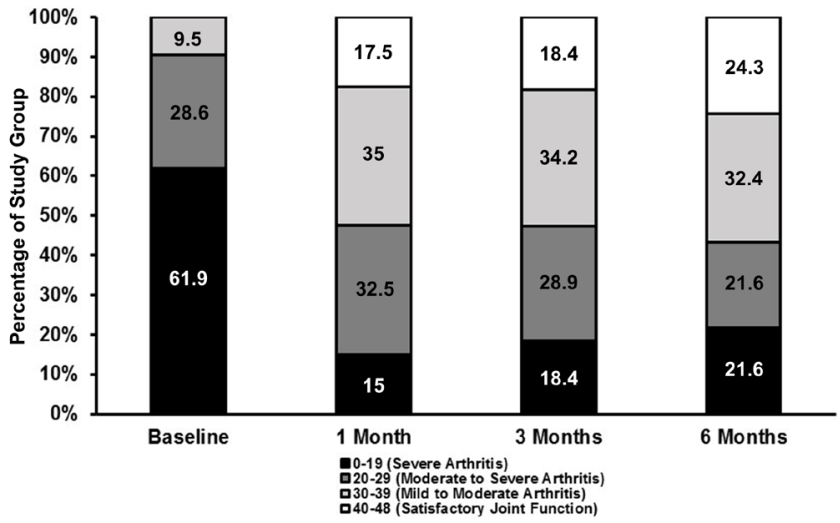

Figure 5 Knee function (Oxford Knee Score) in the cross-over group over time. Baseline, $\mathrm{N}=42 ; 1$ month, $\mathrm{N}=40 ; 3$ months, $\mathrm{N}=38 ; 6$ months, $\mathrm{N}=37$. 
each subject's final visit allowing for comparison to baseline OA status. Fifty-one radiographs were collected, 24 of which were from patients originally treated with CRFA. While most of these patients $(58.3 \% ; 14 / 24)(95 \%$ CI 38.6 to 78.1$)$ had no change in knee OA grade through 12 months, a worsening by one grade was detected in $8.3 \%(2 / 24)(95 \%$ CI 0.0 to 19.4$)$ of the cohort. In the XO group, 27 radiographs were collected 6 months post-CRFA. These images revealed that $81.5 \%(22 / 27)$ (95\% CI 66.8 to 96.1$)$ of grades remained the same as reported at study entry, and worsening by one grade was identified in $7.4 \%(2 / 27)(95 \%$ CI 0.0 to 17.3$)$ of this group. No patients worsened by more than one grade during the study.

\section{Adverse events}

There were 81 AEs that occurred among 42 CRFA patients between 6 and 12 months of the study. Non-SAEs included pain in the index knee (nine events-one of which led to subject discontinuation), with a decision to pursue a surgical alternative $\left(1\right.$; figure $\left.1^{* *}\right)$, pain in the non-index knee (3), musculoskeletal pain (9), and falls (5). SAEs occurred among four patients in the CRFA cohort from 6 to 12 months and included blood/lymphatic (3) and musculoskeletal (1) infections, cardiovascular (1), respiratory (3), gastrointestinal (1), and skin (1) events, and a non-CRFA procedure-related event that involved a musculoskeletal component. None of the SAEs were related to CRFA.

\section{DISCUSSION}

The effect of CRFA to reduce index knee pain by at least $50 \%$ in the majority of the originally treated CRFA study group was sustained at 12 months and validated a portion of the study hypothesis, as $65 \%$ of this cohort experienced this benefit. The mean 4.3-point decrease on the NRS at 12 months compared well with 4.9-point drop that was observed at 6 months. ${ }^{12}$ Patients who elected to have CRFA after originally being treated with an IAS also reported analgesia, as the mean NRS pain score in the XO group fell at least 3.1 points up to 6 months postprocedure, and $49 \%$ of this population had at least $50 \%$ pain relief at 6 months, which confirmed the other portion of the study hypothesis. It is unknown why a difference in response was seen between the originally treated group and the XO group; however, the study was not powered or designed to draw specific conclusions from the XO group and this group should be considered observational given their participation and pathway in the trial. From a functional perspective, after CRFA, the incidence of patients having 'satisfactory joint function' was established and increased throughout the study in both cohorts, while the incidence of patients having 'severe arthritis' diminished with time in both groups. The majority of the originally treated and XO CRFA groups reported 'improved' perceptions of treatment effect on their health at 12 months and across all follow-up visits, respectively. Mean analgesic medication use was similar to baseline at 12 months in the originally treated CRFA group, and no unanticipated AEs occurred as a result of CRFA.

An effect of CRFA on opioid use in this investigation was not detected. As noted in the previous publication, ${ }^{12}$ multiple factors affected our ability to detect a difference in this area, including the duration at which subjects were on opioids prior to the trial, the addictive nature of opioids and the fact that nearly half of the subjects in the CRFA group were taking opioids for reasons beyond their knee pain. However, opioid use stayed consistent with baseline during the trial; therefore, the trial results noted are unlikely to be confounded by these medications.

Interestingly, for the patients described above who were not taking opioids to manage their pain at study baseline $((14 / 67)$ of the original CRFA group), 11 (79\%) patients indicated $\geq 50 \%$ relief of their baseline reported index knee pain at 6 months and their 6.1 mean NRS point reduction was larger than the study wide 4.9-point decrease. Adequately powered studies are warranted to explore the suggestion that CRFA treatment prior to opioid use may be most beneficial to mitigate OA-related knee pain.

As radiographic analysis was not completed through a central lab, assessment variability is to be expected. However, given that less than $9 \%$ of subjects in both CRFA groups experienced OA grade worsening during the study (in similar ratios), a concern that CRFA unreasonably accelerates joint degeneration seems unfounded.

The current treatment algorithm for knee OA has limited effectiveness, and patients often suffer for extended periods before they qualify for TKA. Chronic use of non-steroidal anti-inflammatory drugs can introduce gastrointestinal, cardiovascular, and renal complications ${ }^{18}$; opioids present the risk of tolerance and addiction with escalating dosage over time ${ }^{19}$; physical therapy requires routine visits that increase healthcare expenditures; corticosteroid injections have limited duration of efficacy ${ }^{20}$; viscosupplementation efficacy is equivocal, ${ }^{22} 23$ as is platelet-rich plasma compared with viscosupplementation, ${ }^{24} 25$ and bracing may not be cost-effective. ${ }^{26}$ A TKA is a well-established and successful procedure, ${ }^{12}$ but there are certain populations where one could argue for a more conservative option, ${ }^{27}$ such as when patients are not yet considered 'operative', or have comorbid health issues that would preclude them from surgery or increase the risk profile for undergoing a TKA. A large subgroup of patients who may be not be considered for TKA, such as those with poor glucose control and/or obesity, may become candidates following 6 months to 1 year of mobilization and weight loss afforded by undergoing CRFA first.

This study indicates that large percentages of patients can receive a durable analgesic effect from CRFA, which contrasts with other non-operative treatment options for patients with knee OA. ${ }^{20-25}$ Additionally, at the time of this publication, Santana et al produced the only standard RF knee OA series in the literature providing information to 12 months, ${ }^{7}$ with a mean NRS score reported of 5.8, compared with the mean NRS score of 3.1 in the current CRFA series. While few head-tohead studies exist comparing standard versus cooled radiofrequency directly, such observations are consistent with previous suggestions that the cooling characteristic of CRFA facilitates a larger lesion size than standard $\mathrm{RF}^{28}$ thus, making it more likely that target nerves will be ablated by the CRFA, and perhaps prolonging the time required to complete nerve regeneration. ${ }^{29}$ Further study is needed to examine potential differences between the two technologies.

The beneficial outcomes observed in this current report with respect to CRFA treatment of knee OA extend the bibliography of publications having similar results using CRFA. ${ }^{9-11}$ Our study is the largest prospective randomized comparison to date observing the changes in pain and disability in patients undergoing CRFA. Within this context, the results show that CRFA is safe and durable, thus providing patients who are ineligible for TKA with a seemingly more effective option than IAS, ${ }^{12}$ and perhaps other conservative therapies, 
to gain relief from OA-related knee pain and disability. For those who are TKA candidates, but wish to postpone such a relatively more invasive intervention in favor of CRFA first, evidence suggests benefits of RF-facilitated denervation in this scenario. Taverner et al demonstrated that pulsed RF, but not sham treatment, of patients with painful knees afforded them with a significant pain relief at rest and during exercise prior to total knee joint replacement. ${ }^{30}$ Carli and co-workers used pulsed and thermal RFA to denervate nociceptive nerves of the knee ${ }^{13}$ of a 79 -year-old woman with severe knee OA that required TKA. ${ }^{31}$ The patient had significant knee pain that was unrelieved by opioids, and severely impaired functional activity. The authors attributed significant improvement in the objective and self-reported outcome measures recorded during the 6 weeks of prehabilitation before surgery to the patient's denervation-facilitated preoperative analgesia. The successful prehabilitation was hypothesized to enable rehabilitation implementation post-TKA. The significant gains in functional improvement identified during this study warrant further exploration into this patient population, and large, adequately powered studies (ClinicalTrials.gov identifiers: NCT02746874 and NCT02925442) are in progress to investigate this seemingly purposeful synergistic clinical approach to knee OA between RF-mediated denervation and TKA.

A limitation of this study is the one-way XO option, from IAS to CRFA, but not vice versa. This paradigm is consistent with the intention of the study to test CRFA as a rescue intervention for knee OA, rather than long-standing, conservative IAS. The limitations of this portion of the study are that the remaining IAS group sample size was not large enough to perform statistical test-based comparisons between the originally treated CRFA patients and the IAS group members at 12 months, outcomes of the originally treated CRFA group and those of the XO cohort could not be directly compared at 6 months, because the groups were derived from two different study populations, and an effect of CRFA on opioid use could not be detected, perhaps due to alternate patient conditions that also utilized opioids as therapy. Further, the late addition of the amendment to collect X-rays at the final visit limited our ability to capture data on a large portion of the patients enrolled.

Statistically significant and clinically relevant pain relief and functional improvements were sustained 12 months following CRFA treatment of OA-related knee pain and dysfunction. These effects were reflected by patients' perceptions of their 'improved' health 12 months following CRFA. Moreover, CRFA may rescue patients who have been dissatisfied with results of prior IAS for OA knee pain and who are not candidates for TKA.

\section{Contributors All authors equally contributed in completing this manuscript.}

Funding Financial support was provided to each of the investigator's institutions by Halyard Health to cover the budgeted costs necessary to conduct this study protocol. Data management, study site monitoring and statistics services were performed by a third party, independently of Halyard Health.

Disclaimer This study and its 6-month results were presented as poster and platform abstracts at the American Society of Regional Anesthesia and Pain Medicine (ASRA) 2016 (November) Meeting in San Diego, California, USA, and the American Academy of Pain Medicine (AAPM) 2017 (March) Meeting in Orlando, Florida, USA, and have been published in manuscript form in Regional Anesthesia and Pain Medicine. The 12-month data in this report were presented at the European Society of Regional Anesthesia (ESRA) Congress 2017 in Lugano, Switzerland.

Competing interests TD, DePalma, MD, and LK are paid consultants (clinicaladvisory board) for Halyard Health.

Patient consent for publication Obtained.
Ethics approval This study was approved for engagement by the Western Institutional Review Board (IRB; Puyallup, Washington, USA) and Rush University Medical Center IRB (Chicago, Illinois, USA).

Provenance and peer review Not commissioned; externally peer reviewed.

Open access This is an open access article distributed in accordance with the Creative Commons Attribution Non Commercial (CC BY-NC 4.0) license, which permits others to distribute, remix, adapt, build upon this work non-commercially, and license their derivative works on different terms, provided the original work is properly cited, an indication of whether changes were made, and the use is noncommercial. See: http://creativecommons.org/licenses/by-nc/4.0/.

\section{REFERENCES}

1 Ethgen 0 , Bruyère 0 , Richy $F$, et al. Health-related quality of life in total hip and total knee arthroplasty. A qualitative and systematic review of the literature. J Bone Joint Surg Am 2004;86-A:963-74

2 Crowninshield RD, Rosenberg AG, Sporer SM. Changing demographics of patients with total joint replacement. Clin Orthop Relat Res 2006;443:266-72.

3 Samson AJ, Mercer GE, Campbell DG. Total knee replacement in the morbidly obese: a literature review. ANZ J Surg 2010:80:595-9.

4 Frankel L, Sanmartin C, Conner-Spady B, et al. Osteoarthritis patients' perceptions of "appropriateness" for total joint replacement surgery. Osteoarthritis Cartilage 2012;20:967-73

5 Choi WJ, Hwang SJ, Song JG, et al. Radiofrequency treatment relieves chronic knee osteoarthritis pain: a double-blind randomized controlled trial. Pain 2011;152:481-7.

6 Sarı S, Aydın ON, Turan Y, et al. Which one is more effective for the clinical treatment of chronic pain in knee osteoarthritis: radiofrequency neurotomy of the genicular nerves or intra-articular injection? Int J Rheum Dis 2018;21:1772-8.

7 Santana Pineda MM, Vanlinthout LE, Moreno Martín A, et al. Analgesic effect and functional improvement caused by radiofrequency treatment of genicular nerves in patients with advanced osteoarthritis of the knee until 1 year following treatment. Reg Anesth Pain Med 2017;42:62-8.

8 Kirdemir P, Çatav S, Alkaya Solmaz F. The genicular nerve: radiofrequency lesion application for chronic knee pain. Turk J Med Sci 2017:47:268-72.

9 Bellini M, Barbieri M. Cooled radiofrequency system relieves chronic knee osteoarthritis pain: the first case-series. Anaesthesiol Intensive Ther 2015;47:30-3.

10 McCormick ZL, Korn M, Reddy R, et al. Cooled radiofrequency ablation of the genicular nerves for chronic pain due to knee osteoarthritis: six-month outcomes. Pain Med 2017;18:1631-41.

11 Rojhani S, Qureshi Z, Chhatre A. Water-cooled radiofrequency provides pain relief, decreases disability, and improves quality of life in chronic knee osteoarthritis. Am J Phys Med Rehabil 2017;96:e5-8.

12 Davis T, Loudermilk E, DePalma M, et al. Prospective, multicenter, randomized, crossover clinical trial comparing the safety and effectiveness of cooled radiofrequency ablation with corticosteroid injection in the management of knee pain from osteoarthritis. Reg Anesth Pain Med 2018;43:84-91.

13 Franco CD, Buvanendran A, Petersohn JD, et al. Innervation of the anterior capsule of the human knee: implications for radiofrequency ablation. Reg Anesth Pain Med 2015;40:363-8.

14 Ball RD. The science of conventional and water-cooled monopolar lumbar radiofrequency rhizotomy: an electrical engineering point of view. Pain Physician 2014; 17:e175-211.

15 Dworkin RH, Turk DC, Wyrwich KW, et al. Interpreting the clinical importance of treatment outcomes in chronic pain clinical trials: IMMPACT recommendations. J Pain 2008:9:105-21.

16 Dawson J, Fitzpatrick R, Murray D, et al. Questionnaire on the perceptions of patients about total knee replacement. J Bone Joint Surg Br 1998;80:63-9.

17 Smith MD, Wetherall M, Darby T, et al. A randomized placebo-controlled trial of arthroscopic lavage versus lavage plus intra-articular corticosteroids in the management of symptomatic osteoarthritis of the knee. Rheumatology 2003:42:1477-85.

18 Fine M. Quantifying the impact of NSAID-associated adverse events. Am J Manag Care 2013:19(14 Suppl):s267-72.

19 Trang T, Al-Hasani R, Salvemini D, et al. Pain and poppies: the good, the bad, and the ugly of opioid analgesics. J Neurosci 2015;35:13879-88.

20 Hepper CT, Halvorson JJ, Duncan ST, et al. The efficacy and duration of intra-articular corticosteroid injection for knee osteoarthritis: a systematic review of level I studies. J Am Acad Orthop Surg 2009;17:638-46.

21 Jüni P, Hari R, Rutjes AWS, et al. Intra-articular corticosteroid for knee osteoarthritis. Cochrane Database Syst Rev 2015;28.

22 Jevsevar D, Donnelly P, Brown GA, et al. Viscosupplementation for osteoarthritis of the knee: a systematic review of the evidence. J Bone Joint Surg Am 2015;97:2047-60.

23 Strand V, McIntyre LF, Beach WR, et al. Safety and efficacy of US-approved viscosupplements for knee osteoarthritis: a systematic review and meta-analysis of randomized, saline-controlled trials. J Pain Res 2015;8:217-28. 


\section{Original article}

24 Filardo G, Di Matteo B, Di Martino A, et al. Platelet-rich plasma intra-articular knee injections show no superiority versus Viscosupplementation: a randomized controlled trial. Am J Sports Med 2015;43:1575-82.

25 Meheux CJ, McCulloch PC, Lintner DM, et al. Efficacy of intra-articular plateletrich plasma injections in knee osteoarthritis: a systematic review. Arthroscopy 2016:32:495-505.

26 Woods B, Manca A, Weatherly $H$, et al. Cost-effectiveness of adjunct nonpharmacological interventions for osteoarthritis of the knee. PLoS One 2017;12:e0172749.

27 Hochberg MC, Altman RD, April KT, et al. American College of rheumatology 2012 recommendations for the use of nonpharmacologic and pharmacologic therapies in osteoarthritis of the hand, hip, and knee. Arthritis Care Res 2012;64:465-74.
28 Watanabe I, Masaki R, Min N, et al. Cooled-tip ablation results in increased radiofrequency power delivery and lesion size in the canine heart: importance of catheter-tip temperature monitoring for prevention of popping and impedance rise. J Interv Card Electrophysiol 2002;6:9-16.

29 Choi EJ, Choi YM, Jang EJ, et al. Neural ablation and regeneration in pain practice. Korean J Pain 2016:29:3-11.

30 Taverner MG, Ward TL, Loughnan TE. Transcutaneous pulsed radiofrequency treatment in patients with painful knee awaiting total knee joint replacement. Clin J Pain 2010;26:429-32.

31 Carli F, Chora D, Awasthi R, et al. Preoperative pulse and thermal radiofrequency facilitates prehabilitation and subsequent rehabilitation of a patient scheduled for total knee arthroplasty. Can J Anesth/J Can Anesth 2015;62:1355-6. 